\title{
The Global Stability of 2-D Subsonic Circulatory Flows for the Steady Isothermal Gas
}

\author{
Dacheng Cui ${ }^{1}$ \\ ${ }^{1}$ School of Information Engineering, Nanjing Xiaozhuang University, Nanjing, P.R.China \\ Correspondence: Dacheng Cui, School of Information Engineering, Nanjing Xiaozhuang University, Nanjing 211171, \\ P.R.China. E-mail: dchcui@163.com
}

Received: October 1, 2017 Accepted: October 31, 2017 Online Published: November 17, 2017

doi:10.5539/jmr.v9n6p148 URL: https://doi.org/10.5539/jmr.v9n6p148

The research is financed by Jiangsu Province University Natural Science Research Project (No.14KJB110018)

\begin{abstract}
This paper is a complement of our work in (Cui \& Li, 2011) where we have established the global subsonic circulatory solution for the polytropic gas. In this paper, we are concerned with the global stability of the 2-D subsonic circulatory flow around a perturbed circular body for the isothermal gas. The flow is assumed to be isothermal, isentropic, irrotational and described by a steady Euler equations, which can be reduced into a second order quasilinear elliptic equation in a exterior domain with suitable physical conditions. The unique existence and the state of the flow at infinity are obtained under nature physical assumption.
\end{abstract}

Keywords: Euler equations, isothermal gas, subsonic circulatory flow, weighted Hölder estimates.

Mathematical Subject Classification 2000: 35L70, 35L65, 35L67, 76N15.

\section{Introduction}

We are concerned with the global stability problem of a 2-D perturbed subsonic circulatory flow for the isothermal gas. In (Courant \& Friedrichs, 1948) the special subsonic circulatory flows are constructed when the obstacle is regular circular body. If the obstacle is suitably perturbed, the global subsonic circulatory flow is stable? L.Bers (see Bers, 1945) uses the pesudo-complex analysis method to obtain the global existence of the subsonic circulatory flow under the conditions that the adiabatic exponent $\gamma=-1$. In (Cui \& Li, 2011), we have established the global existence of the subsonic circulatory flows solutions for inviscid gases with adiabatic exponent $1<\gamma<3$. In the present paper, our goal is to establish the global existence and stability of subsonic circulatory flows solution for the subsonic isothermal gas around a perturbed circular body. The so-called isothermal gas means that the pressure $P$ and the density $\rho$ of gas are described by the state equation $P=A \rho$ for some constant $A>0$ (see Cui \& Yin, 2007 and the references therein). In this case, the sound speed is a constant independent of the density $\rho$.

We will use 2-D steady isentropic Euler system to describe the motion of gas

$$
\left\{\begin{array}{l}
\partial_{1}\left(\rho u_{1}\right)+\partial_{2}\left(\rho u_{2}\right)=0, \\
\partial_{1}\left(P+\rho u_{1}^{2}\right)+\partial_{2}\left(\rho u_{1} u_{2}\right)=0, \\
\partial_{1}\left(\rho u_{1} u_{2}\right)+\partial_{2}\left(P+\rho u_{2}^{2}\right)=0,
\end{array}\right.
$$

where $\rho>$ is the density, $u=\left(u_{1}, u_{2}\right)$ is the velocity, and $P=P(\rho)$ is the pressure of the flow. For the isothermal gas, the state equation is $P=A \rho, A>0$ is a fixed constant. In this case, $c(\rho)=\sqrt{A}$ is the local sound speed.

In addition, we assume the gas is irrotational, that is

$$
\partial_{x_{2}} u_{1}=\partial_{x_{1}} u_{2}
$$

As in (Courant \& Friedrichs, 1948), the last two equations in (1) together (2) yield the Bernoulli's law:

$$
\frac{1}{2}|u|^{2}+A \ln \rho=B,
$$

here the term $A \ln \rho$ is the specific enthalpy for the isothermal gas and $B$ is the Bernoulli' constant.

We now give a mathematical description on the subsonic circulatory flows for the isothermal gas around the circular body $\left\{\left(x_{1}, x_{2}\right): r=\sqrt{x_{1}^{2}+x_{2}^{2}} \leq 1+\delta(x)\right\}$. Set $\Omega=\{r: r>1+\delta(x)\}$ with $\delta(x)$ satisfies

$$
\operatorname{supp}(\delta(x)) \subset\left\{x: \frac{2}{3} \leq r \leq \frac{3}{2}\right\}, \quad\|\delta(x)\|_{C^{2, \alpha}\left(\mathbb{R}^{2}\right)} \leq \varepsilon,
$$


for some $\alpha \in(0,1)$ and $\varepsilon \in(0,1)$.

The solid wall $\partial \Omega$ is assumed to be impermeable

$$
\left(u_{1}, u_{2}\right) \cdot \vec{v}=0 \quad \text { on } \quad \partial \Omega,
$$

where $\vec{v}$ stands for the unit exterior normal to the boundary $\partial \Omega$.

As in (Cui \& Li, 2011), through application of Green formula on the first equation in (1), the mass-flux of the circulatory flow should be invariant along each radial ray $l$ which starts from the boundary $\partial \Omega$, so the flow should satisfy the following generalized mass-flux condition

$$
\lim _{r \rightarrow \infty} \frac{1}{\ln r} \int_{l}\left(\rho u_{1}, \rho u_{2}\right) \cdot \vec{n} d s=e^{\frac{B}{A}}
$$

here $\vec{n}$ is the out normal of $l$.

Based on the first equation in (1) and the condition (4), we can introduced a stream function $\psi$ such that

$$
\psi_{x_{1}}=\rho u_{2}, \quad \psi_{x_{2}}=-\rho u_{1}, \quad \text { with } \quad \psi=m \text { on } \partial \Omega,
$$

here $m$ is some fixed constant.

Thus the irrotationality can be written as $\operatorname{div}\left(\frac{1}{\rho} \nabla \psi\right)=0$. Notice that from the Bernoulli's law we can solve

$$
\partial_{1} \rho=\frac{1}{\rho(2 B-2 A \ln \rho-A)}\left(\partial_{1} \psi \partial_{11}^{2} \psi+\partial_{2} \psi \partial_{12}^{2} \psi\right), \quad \partial_{2} \rho=\frac{1}{\rho(2 B-2 A \ln \rho-A)}\left(\partial_{1} \psi \partial_{12}^{2} \psi+\partial_{2} \psi \partial_{22}^{2} \psi\right),
$$

with the term $\rho(2 B-2 A \ln \rho-A)=\rho\left(\left|\frac{\nabla \psi}{\rho}\right|^{2}-A\right)<0$ holds for the subsonic flow.

Therefore, if $\psi \in C^{2}(\Omega) \cap C^{0}(\bar{\Omega})$, the nonlinear problem (1)-(5) is equivalent to

$$
\left\{\begin{array}{l}
\left(\left(\partial_{2} \psi\right)^{2}-A \rho^{2}\right) \partial_{11}^{2} \psi-2 \partial_{1} \psi \partial_{2} \psi \partial_{12}^{2} \psi+\left(\left(\partial_{1} \psi\right)^{2}-A \rho^{2}\right) \partial_{22}^{2} \psi=0, \quad \text { in } \Omega, \\
\frac{1}{2}|\nabla \psi|^{2}+A \rho^{2} \ln \rho=B \rho^{2}, \quad \text { in } \Omega, \\
\psi=m, \quad \text { on } \partial \Omega, \\
\lim _{|x| \rightarrow+\infty} \frac{\psi(x)}{\ln |x|}=e^{\frac{B}{A}}
\end{array}\right.
$$

We can get the circulatory subsonic solution $\left(\psi_{0}(r), \rho_{0}(r)\right)$ of the system (7) in the domain $\Omega_{0}=\{x:|x|>1\}$ by the analogous methods as in (Cui \& Li, 2011), with each streamline being a circle and the center being at the origin just as illustrated in (Courant \& Friedrichs, 1948). For the specific details, one can see the appendix in this paper.

$$
\rho_{0}(r)=\exp \left(\frac{1}{A}\left(B-\frac{1}{2 r^{2}}\right)\right), \quad \psi_{0}(r)=\int_{1}^{r} \rho_{0}(s) \frac{1}{s} d s+m
$$

Remark 1.1 From the subsonic property of $\left(\psi_{0}(r), \rho_{0}(r)\right)$ in the domain $\Omega_{0}$, there exits a constant $r_{0} \in\left(\frac{1}{2}, 1\right)$ and a small constant $c_{0}>0$ such that

$$
A>\frac{1}{r_{0}^{2}}+c_{0}
$$

then the background solution $\left(\psi_{0}(r), \rho_{0}(r)\right)$ given by (8) can be extended into $\left[r_{0}, \infty\right)$ which also satisfy the system $(7)$ in the domain $\mathbb{R}^{2} \backslash B_{r_{0}}(0)$.

Our main result in this paper is

Theorem 1.1 There exists a small constant $\varepsilon_{0}>0$ which is dependent on $A$ and $B$, such that for any $\varepsilon<\varepsilon_{0}$, the problem (7) has a unique circulatory subsonic solution $(\psi, \rho) \in C^{2, \alpha}(\bar{\Omega}) \times C^{1, \alpha}(\bar{\Omega})$. Moreover, $\left|\nabla\left(\psi(x)-\psi_{0}(r)\right)\right|$ and $\left|\rho(x)-\rho_{0}(r)\right|$ tend to zero as $|x| \rightarrow \infty$ with decay rates $|x|^{-2}$ and $|x|^{-3}$ respectively.

Remark 1.2 From the physical point of view (see Bers, 1958; Finn \& Gilbarg, 1957), the subsonic circulatory flow solution in Theorem 1.1 is stable, since it satisfies $\lim _{|x| \rightarrow \infty} u(x)=(0,0)$. 


\section{The Reformulation on Problem (7)}

As in (Cui \& Li, 2011), it is convenient to use the coordinates transformation: $z_{1}=x_{1}(1+\delta(x))^{-1}, z_{2}=x_{2}(1+\delta(x))^{-1}$. In this case, the exterior domain $\Omega$ is changed into $\Omega_{0}$ and $\partial_{1}=\left(1+o_{11}(z)\right) \partial_{z_{1}}+o_{12}(z) \partial_{z_{2}}, \partial_{2}=o_{21}(z) \partial_{z_{1}}+\left(1+o_{22}(z)\right) \partial_{z_{2}}$.

$$
\begin{gathered}
o_{i j}(z)=-\frac{\delta(x)}{1+\delta(x)} \delta_{i j}-\frac{x_{j} \partial_{i} \delta(x)}{(1+\delta(x))^{2}} ; \\
\operatorname{supp}\left(o_{i j}(z)\right) \subset\left\{x: \frac{1}{2} \leq|x| \leq \frac{3}{2}\right\}, \quad\left|o_{i j}(z)\right|_{1, \alpha}^{(3)} \leq C \varepsilon, \quad i, j=1,2 .
\end{gathered}
$$

By a tedious computation, the first two equation in (7) can be written as respectively

$$
\left(\left(\partial_{z_{2}} \psi\right)^{2}-A \rho^{2}\right) \partial_{z_{1}}^{2} \psi-2 \partial_{z_{1}} \psi \partial_{z_{2}} \psi \partial_{z_{1} z_{2}}^{2} \psi+\left(\left(\partial_{z_{1}} \psi\right)^{2}-A \rho^{2}\right) \partial_{z_{2}}^{2} \psi=G_{1}\left(z, \nabla_{z} \psi, \rho\right)
$$

and

$$
\frac{1}{2}\left|\nabla_{z} \psi\right|^{2}+A \rho^{2} \ln \rho=B \rho^{2}-G_{2}\left(z, \nabla_{z} \psi\right)
$$

where

$$
\begin{gathered}
G_{1}\left(z, \nabla_{z} \psi, \rho\right)=O\left(\sum_{i=1}^{2} \sum_{j=1}^{2}\left(\left|o_{i j}(z)\right|+\left|\nabla_{z} o_{i j}(z)\right|\right)\left(\left|\nabla_{z} \psi\right|^{3}+\left|\nabla_{z} \psi\right|^{2}\left|\nabla_{z}^{2} \psi\right|+\rho^{2}\left|\nabla_{z} \psi\right|+\rho^{2}\left|\nabla_{z}^{2} \psi\right|\right)\right), \\
G_{2}\left(z, \nabla_{z} \psi\right)=\frac{1}{2}\left(o_{11}^{2}(z)+2 o_{11}(z)+o_{21}^{2}(z)\right)\left(\partial_{z 1} \psi\right)^{2}+\left(\left(1+o_{11}(z)\right) o_{12}(z)+o_{21}(z)\left(1+o_{22}(z)\right)\right) \partial_{z 1} \psi \partial_{z_{2}} \psi \\
+\frac{1}{2}\left(o_{12}^{2}(z)+2 o_{22}(z)+o_{22}^{2}(z)\right)\left(\partial_{z_{2}} \psi\right)^{2} .
\end{gathered}
$$

Using the notations $\Phi=\psi-\psi_{0}$ and $\Psi=\rho-\rho_{0}$, then the nonlinear problem (7) can be changed into

$$
\left\{\begin{array}{l}
L(\Phi)=\Delta \Phi-\left(\sum_{i=1}^{2} \sum_{j=1}^{2} a_{i j}(z) \partial_{i j}^{2} \Phi+\sum_{i=1}^{2} b_{i}(z) \partial_{i} \Phi\right)=-\frac{1}{A \rho_{0}^{2}} F_{1}(z, \nabla \Phi, \Psi), \quad \text { in } \Omega_{0}, \\
\Psi=\frac{1}{\Pi(z)}\left(\partial_{1} \psi_{0} \partial_{1} \Phi+\partial_{2} \psi_{0} \partial_{2} \Phi\right)+F_{2}(z, \nabla \Phi, \Psi), \quad \text { in } \Omega_{0}, \\
\Phi=0, \text { on }|z|=1, \\
\lim _{|z| \rightarrow \infty} \frac{\Phi(z)}{\ln |z|}=0
\end{array}\right.
$$

where

$$
\begin{aligned}
& a_{11}(z)=\frac{\left(\partial_{2} \psi_{0}\right)^{2}}{A \rho_{0}^{2}}=\frac{z_{2}^{2}}{A r^{4}}, \\
& a_{12}(z)=-\frac{\partial_{1} \psi_{0} \partial_{2} \psi_{0}}{A \rho_{0}^{2}}=-\frac{z_{1} z_{2}}{A r^{4}}, \\
& a_{22}(z)=\frac{\left(\partial_{1} \psi_{0}\right)^{2}}{A \rho_{0}^{2}}=\frac{z_{1}^{2}}{A r^{4}}, \\
& b_{1}(z)=\frac{2\left(\partial_{1} \psi_{0} \partial_{22}^{2} \psi_{0}-\partial_{2} \psi_{0} \partial_{12}^{2} \psi_{0}\right)}{A \rho_{0}^{2}}-\frac{2 \partial_{1} \psi_{0} \Delta \psi_{0}}{\rho_{0} \Pi(z)}=\frac{2 z_{1}}{r^{2}\left(A r^{2}-1\right)}, \\
& b_{2}(z)=\frac{2\left(\partial_{2} \psi_{0} \partial_{11}^{2} \psi_{0}-\partial_{1} \psi_{0} \partial_{12}^{2} \psi_{0}\right)}{A \rho_{0}^{2}}-\frac{2 \partial_{2} \psi_{0} \Delta \psi_{0}}{\rho_{0} \Pi(z)}=\frac{2 z_{2}}{r^{2}\left(A r^{2}-1\right)},
\end{aligned}
$$

and

$$
\begin{aligned}
F_{1}(z, \nabla \Phi, \Psi)= & G_{1}(z, \nabla \psi, \rho)+G_{4}(z, \nabla \Phi, \Psi), \\
F_{2}(z, \nabla \Phi, \Psi)= & \left.G_{2}(z, \nabla \psi)+G_{3}(z, \nabla \Phi, \Psi)\right), \\
G_{3}(z, \nabla \Phi, \Psi)= & \frac{1}{2}|\nabla \Phi|^{2}-B \Psi^{2}+A\left(\rho^{2} \ln \rho-\rho_{0}^{2} \ln \rho_{0}-\rho_{0}\left(2 \ln \rho_{0}+1\right) \Psi\right), \\
G_{4}(z, \nabla \Phi, \Psi)= & A \Delta \psi\left(\Psi^{2}+2 \rho_{0} F_{1}(z, \nabla \Phi, \Psi)\right)+\frac{2 A \rho_{0}}{\Pi(z)} \Delta \Phi\left(\partial_{1} \psi_{0} \partial_{1} \Phi+\partial_{2} \psi_{0} \partial_{2} \Phi\right) \\
& -\partial_{11}^{2} \psi\left(\partial_{2} \Phi\right)^{2}-\partial_{22}^{2} \psi\left(\partial_{1} \Phi\right)^{2}+2 \partial_{12}^{2} \psi \partial_{1} \Phi \partial_{2} \Phi+2 \partial_{1} \psi_{0} \partial_{2} \Phi \partial_{12}^{2} \Phi \\
& -2 \partial_{1} \psi_{0} \partial_{1} \Phi \partial_{22}^{2} \Phi+2 \partial_{2} \psi_{0} \partial_{1} \Phi \partial_{12}^{2} \Phi-2 \partial_{2} \psi_{0} \partial_{2} \Phi \partial_{11}^{2} \Phi
\end{aligned}
$$


here $\Pi(z) \equiv \rho_{0}\left(2 B-2 A \ln \rho_{0}-A\right)=\rho_{0}\left(\frac{1}{r^{2}}-A\right)<0$.

We introduce some weighted Hölder space and corresponding norms which have been used in (Gilbarg \& Tudinger, 1998; Chen, 2009; Cui \& Li, 2011 and so on). For $x, y \in \Omega_{0}$, let us write $r_{x}=|x|, r_{y}=|y|$ and $r_{x y}=\min \left(r_{x}, r_{y}\right)$. For $k \in \mathbb{Z}^{+} \cup\{0\}$, $0<\alpha<1, l \in \mathbb{R}$ and $u \in C^{k, \alpha}\left(\bar{\Omega}_{0}\right)$, we define

$$
|u|_{k, 0}^{(l)}=\sum_{j=0}^{k} \sup _{x \in D_{0},|\beta|=j} r_{x}^{j+l}\left|D^{\beta} u(x)\right|, \quad|u|_{k, \alpha}^{(l)}=|u|_{k, 0}^{(l)}+\sup _{x, y \in D_{0},|\beta|=k} r_{x, y}^{k+l+\alpha} \frac{\left|D^{\beta} u(x)-D^{\beta} u(y)\right|}{|x-y|^{\alpha}} .
$$

and the corresponding function space is defined as

$$
H_{k, \alpha}^{(l)}=\left\{u \in C^{k, \alpha}\left(\bar{\Omega}_{0}\right):|u|_{k, \alpha}^{(l)}<+\infty\right\} .
$$

Based on above, Theorem 1.1 follows the following conclusion:

Theorem 2.1 There exists some positive constants $\varepsilon_{0}, C$ depending on $A, B$, such that for any $\varepsilon<\varepsilon_{0}$, the problem (15) has a unique global solution $(\Phi, \Psi) \in C^{2, \alpha}\left(\bar{\Omega}_{0}\right) \times C^{1, \alpha}\left(\bar{\Omega}_{0}\right)$ with the following estimate

$$
|\Phi|_{2, \alpha}^{(0)}+|\nabla \Phi|_{1, \alpha}^{(2)}+|\Psi|_{1, \alpha}^{(3)} \leq C \varepsilon
$$

where $0<\alpha<1$.

\section{The Proof of the Theorem 2.1}

In order to solve the nonlinear problem (15), a quasilinear elliptic boundary value problem on the unbounded domain $\Omega_{0}$ coupled with a algebraic equation, the key is to establish the uniform weighted Hölder estimate. As in (Cui \& Li, 2011; Gilbarg \& Tudinger, 1998), based on this estimate we can use the continuity method to solve the linearized problem of (15). Furthermore, by this estimate together with the standard fixed-point argument in (Gilbarg \& Tudinger, 1998), we can arrive at the existence and uniqueness of the solution of the nonlinear problem (15). So we need study the following problem for any $\sigma \in[0,1]$ :

$$
\left\{\begin{array}{l}
L_{\sigma}\left(\Phi_{\sigma}\right)=\Delta \Phi_{\sigma}-\sigma\left(\sum_{i=1}^{2} \sum_{j=1}^{2} a_{i j}(z) \partial_{i j}^{2} \Phi_{\sigma}+\sum_{i=1}^{2} b_{i}(z) \partial_{i} \Phi_{\sigma}\right) \\
\equiv \partial_{r}^{2} \Phi_{\sigma}+\frac{1}{r} \partial_{r} \Phi_{\sigma}+\frac{1}{r^{2}} \partial_{\theta}^{2} \Phi_{\sigma}+\sigma c_{1}(r) \frac{1}{r} \partial_{r} \Phi_{\sigma}+\sigma c_{2}(r) \frac{1}{r^{2}} \partial_{\theta}^{2} \Phi_{\sigma} \\
\quad=-\frac{1}{A \rho_{0}^{2}(r)} F_{1}(z, \nabla \Phi, \Psi), \text { in } \Omega_{0}, \\
\Psi_{\sigma}=\frac{1}{\Pi(z)}\left(\partial_{1} \psi_{0} \partial_{1} \Phi_{\sigma}+\partial_{2} \psi_{0} \partial_{2} \Phi_{\sigma}\right)+F_{2}(z, \nabla \Phi, \Psi), \text { in } \Omega_{0}, \\
\Phi_{\sigma}=0, \quad \text { on }|r|=1, \\
\lim _{|z| \rightarrow \infty} \frac{\Phi_{\sigma}(z)}{\ln |z|}=0 .
\end{array}\right.
$$

with $c_{1}(r)=-\frac{3 A-\frac{1}{r^{2}}}{A\left(A-\frac{1}{r^{2}}\right)} \frac{1}{r^{2}}<0, c_{2}(r)=-\frac{1}{A r^{2}}$.

From the subsonic property (9) together with (16), we have

$$
0<c_{0} A^{-1}|\xi|^{2} \leq\left(1-\frac{1}{A r_{0}^{2}}\right)|\xi|^{2} \leq|\xi|^{2}-\sigma \sum_{i=1}^{2} \sum_{j=1}^{2} a_{i j}(z) \xi_{i} \xi_{j} \leq|\xi|^{2}, \forall z \in \Omega_{0}, \xi \in \mathbb{R}^{2} \backslash\{0\}, \sigma \in[0,1],
$$

and

$$
\sum_{i=1}^{2} \sum_{j=1}^{2}\left|a_{i j}\right|_{0, \alpha}^{(2)}+\sum_{i=1}^{2}\left|b_{i}\right|_{1, \alpha}^{(3)} \leq C
$$

With respect to the source terms $F_{1}(z, \nabla \Phi, \Psi), F_{2}(z, \nabla \Phi, \Psi)$ in (19), we give the detailed estimates as following: 
Lemma 3.1 For $(\nabla \psi, \rho) \in H_{1, \alpha}^{(1)} \times H_{1, \alpha}^{(0)}$, then we have

$$
\begin{aligned}
& \left|G_{1}\left(z, \nabla_{z} \psi, \rho\right)\right|_{0, \alpha}^{(4)} \leq C \varepsilon\left(\left(|\rho|_{0, \alpha}^{(0)}\right)^{2}+\left(\left|\nabla_{z} \psi\right|_{1, \alpha}^{(1)}\right)^{2}\right)\left|\nabla_{z} \psi\right|_{1, \alpha}^{(1)} ; \\
& \left|G_{2}\left(z, \nabla_{z} \psi\right)\right|_{1, \alpha}^{(3)} \leq C \varepsilon\left(\left|\nabla_{z} \psi\right|_{1, \alpha}^{(1)}\right)^{2} \\
& \left|F_{1}(z, \nabla \Phi, \Psi)\right|_{0, \alpha}^{(4)} \leq C \varepsilon\left(\left(|\rho|_{0, \alpha}^{(0)}\right)^{2}+\left(\left|\nabla_{z} \psi\right|_{1, \alpha}^{(1)}\right)^{2}\right)\left|\nabla_{z} \psi\right|_{1, \alpha}^{(1)}+C\left(1+\left|\nabla_{z} \psi\right|_{1, \alpha}^{(1)}\right)\left(\left(|\nabla \Phi|_{1, \alpha}^{(1)}\right)^{2}+\left(|\Psi|_{0, \alpha}^{(1)}\right)^{2}\right) ; \\
& \left|F_{2}(z, \nabla \Phi, \Psi)\right|_{1, \alpha}^{(3)} \leq C\left(\left(|\nabla \Phi|_{1, \alpha}^{(2)}\right)^{2}+\left(|\Psi|_{1, \alpha}^{(2)}\right)^{2}+\varepsilon\left(\left|\nabla_{z} \psi\right|_{1, \alpha}^{(1)}\right)^{2}\right) ; \\
& \left|F_{1}\left(z, \nabla \Phi_{1}, \Psi_{1}\right)-F_{2}\left(z, \nabla \Phi_{2}, \Psi_{2}\right)\right|_{0, \alpha}^{(4)} \leq C\left(\varepsilon \sum_{j=1}^{2}\left(\left(\left|\rho_{j}\right|_{1, \alpha}^{(0)}\right)^{2}+\left|\rho_{j}\right|_{1, \alpha}^{(0)}+\left(\left|\nabla_{z} \psi_{j}\right|_{1, \alpha}^{(1)}\right)^{2}\right)\right. \\
& \left.\quad+\sum_{k=1}^{2} \sum_{l=1}^{2}\left(1+\left|\nabla_{z} \psi_{k}\right|_{1, \alpha}^{(1)}\right)\left(\left|\nabla \Phi_{l}\right|_{1, \alpha}^{(1)}+\left|\Psi_{l}\right|_{1, \alpha}^{(2)}\right)\right)\left(\left|\nabla_{z}\left(\Phi_{1}-\Phi_{2}\right)\right|_{1, \alpha}^{(1)}+\left|\Psi_{1}-\Psi_{2}\right|_{1, \alpha}^{(2)}\right) ; \\
& \left|F_{2}\left(z, \nabla \Phi_{1}, \Psi_{1}\right)-F_{1}\left(z, \nabla \Phi_{2}, \Psi_{2}\right)\right|_{1, \alpha}^{(3)} \leq C\left(\sum_{j=1}^{2}\left(\left|\nabla \Phi_{j}\right|_{1, \alpha}^{(2)}+\left|\Psi_{j}\right|_{1, \alpha}^{(2)}+\varepsilon\left|\nabla_{z} \psi_{j}\right|_{1, \alpha}^{(1)}\right)\right)\left(\left|\nabla\left(\Phi_{1}-\Phi_{2}\right)\right|_{1, \alpha}^{(1)}+\left|\Psi_{1}-\Psi_{2}\right|_{1, \alpha}^{(2)}\right) .
\end{aligned}
$$

Proof. The Lemma is only a direct computation which we omit, using (13), (14), (17) and (10).

Now we try to obtain the weighted Hölder norm of the solution to (19), which is motivated by Lemma 6.20 in (Gilbarg \& Tudinger, 1998). In the following, by the separation variable method as in (Cui \& $\mathrm{Li}, 2011 ; \mathrm{Li}, \mathrm{Xu} \&$ Yin, 2015), the estimate of the infinity state of the solution to (19) is established. This together with the weighted Hölder estimate in (Gilbarg \& Tudinger, 1998), we can get the uniform weighted Hölder estimate.

Lemma 3.2 (Weighted Hölder estimate) For any given $(\Phi, \Psi) \in C^{2, \alpha}\left(\bar{\Omega}_{0}\right) \times C^{1, \alpha}\left(\bar{\Omega}_{0}\right)$, if $\Phi_{\sigma} \in C^{2}\left(\Omega_{0}\right) \cap C\left(\bar{\Omega}_{0}\right)$ is a solution of the problem (19) for any $\sigma \in[0,1]$, then there exists a generic constant $C>0$ independent of $\sigma$ and $\delta$ such that

$$
\left|\Phi_{\sigma}\right|_{2, \alpha}^{(0)}+\left|\nabla \Phi_{\sigma}\right|_{1, \alpha}^{(2)}+\left|\Psi_{\sigma}\right|_{1, \alpha}^{(3)} \leq C\left(\left|F_{1}(z, \nabla \Phi, \Psi)\right|_{0, \alpha}^{(3)}+\left|F_{2}(z, \nabla \Phi, \Psi)\right|_{1, \alpha}^{(3)}\right) .
$$

where the generic constant $C>0$ is independent of $\sigma, \delta$.

Proof. We firstly establish the $L^{\infty}$ bound by the separation variable method as in (Cui \& Li, 2011). Set

$$
\Phi_{\sigma}(r, \theta)=R_{0}(r)+\sum_{n=1}^{\infty}\left(R_{n}^{1}(r) \cos (n \theta)+R_{n}^{2}(r) \sin (n \theta)\right),
$$

then by (19) we can solve

where

$$
\begin{gathered}
R_{0}^{\prime \prime}(r)+\left(1+\sigma c_{1}(r)\right) \frac{1}{r} R_{0}^{\prime}(r)=f_{0}(r), \quad R_{0}(1)=0, \quad \lim _{r \rightarrow \infty} \frac{R_{0}(r)}{\ln r}=0 \\
\left(R_{n}^{i}\right)^{\prime \prime}(r)+\frac{1}{r}\left(R_{n}^{i}\right)^{\prime}(r)-\frac{n^{2}}{r^{2}} R_{n}^{i}(r)=f_{n}^{i}(r), \quad R_{n}^{i}(1)=0, \quad \lim _{r \rightarrow \infty} \frac{R_{n}^{i}(r)}{\ln r}=0,
\end{gathered}
$$

$$
\begin{aligned}
& f_{0}(r)=-\frac{1}{2 \pi A \rho_{0}^{2}(r)} \int_{0}^{2 \pi} F_{1}(z, \nabla \Phi, \Psi) d \theta \\
& f_{n}^{1}(r)=\frac{1}{\pi} \int_{0}^{2 \pi}\left(-\frac{1}{A \rho_{0}^{2}(r)} F_{1}(z, \nabla \Phi, \Psi)+\sigma\left(c_{1}(r) \frac{1}{r} \partial_{r} \Phi_{\sigma}+c_{2}(r) \frac{1}{r^{2}} \partial_{\theta}^{2} \Phi_{\sigma}\right)\right) \cos (n \theta) d \theta \\
& f_{n}^{2}(r)=\frac{1}{\pi} \int_{0}^{2 \pi}\left(-\frac{1}{A \rho_{0}^{2}(r)} F_{1}(z, \nabla \Phi, \Psi)+\sigma\left(c_{1}(r) \frac{1}{r} \partial_{r} \Phi_{\sigma}+c_{2}(r) \frac{1}{r^{2}} \partial_{\theta}^{2} \Phi_{\sigma}\right)\right) \sin (n \theta) d \theta
\end{aligned}
$$

Then $R_{0}(r)$ and $R_{n}^{i}(r)(n \geq 1 ; i=1,2)$ have the expressions as

$$
\begin{aligned}
& R_{0}(r)=-\int_{1}^{r} e^{-\int_{1}^{y} c_{3}(s) d s} \int_{y}^{\infty} e^{\int_{1}^{l} c_{3}(s) d s} f_{0}(l) d l d y \\
& R_{n}^{i}(r)=\frac{1}{2 n} r^{-n} \int_{1}^{+\infty} s^{-n+1} f_{n}^{i}(s) d s-\frac{1}{2 n} r^{n} \int_{r}^{+\infty} s^{-n+1} f_{n}^{i}(s) d s-\frac{1}{2 n} r^{-n} \int_{1}^{r} s^{n+1} f_{n}^{i}(s) d s .
\end{aligned}
$$


By the expressions of $c_{i}(r)(i=1,2)$ and the subsonic property in (9), one has

$$
c_{3}(r)=\left(1+\sigma c_{1}(r)\right) \frac{1}{r}, \quad 0<\frac{c_{4}}{r} \leq e^{-\int_{1}^{r} c_{3}(s) d s} \leq \frac{c_{5}}{r},
$$

here $c_{4}$ and $c_{5}$ are positive constants independent of $\sigma$ and $\delta$.

From (24)-(26), we have the following estimates

$$
\left\{\begin{array}{l}
\left|R_{0}(r)\right| \leq C\left|f_{0}\right|_{0,0}^{(3)} \leq C\left|F_{1}(z, \nabla \Phi, \Psi)\right|_{0,0}^{(3)}, \\
\left|R_{0}(r)+\int_{1}^{\infty} e^{-\int_{1}^{r} c_{3}(s) d s} \int_{r}^{\infty} e^{\int_{1}^{l} c_{3}(s) d s} f_{0}(l) d l d r\right| \leq C\left|F_{1}(z, \nabla \Phi, \Psi)\right|_{0,0}^{(3)} r^{-1}, \\
\left|R_{n}^{i}(r)\right| \leq \frac{C}{n^{2}}\left|f_{n}^{i}\right|_{0,0}^{(3)} r^{-1} \leq \frac{C}{n^{2}}\left(\left|F_{1}(z, \nabla \Phi, \Psi)\right|_{0,0}^{(3)}+\sigma r^{-1}\left|\nabla \Phi_{\sigma}\right|_{1,0}^{(1)}\right) r^{-1}
\end{array}\right.
$$

Finally, combing (21) with (27) yields the following $L^{\infty}$ estimates:

$$
\left\{\begin{array}{l}
\left|\Phi_{\sigma}(z)\right| \leq C\left(\left|F_{1}(z, \nabla \Phi, \Psi)\right|_{0,0}^{(3)}+\sigma\left|\nabla \Phi_{\sigma}\right|_{1,0}^{(1)} r^{-2}\right), \\
\left|\Phi_{\sigma}(z)+\int_{1}^{\infty} e^{-\int_{1}^{r} c_{3}(s) d s} \int_{r}^{\infty} e^{\int_{1}^{l} c_{3}(s) d s} f_{0}(l) d l d r\right| \leq C\left(\left|F_{1}(z, \nabla \Phi, \Psi)\right|_{0,0}^{(3)}+\sigma\left|\nabla \Phi_{\sigma}\right|_{1,0}^{(1)} r^{-1}\right) r^{-1},
\end{array}\right.
$$

As in (Cui \& Li, 2011; Gilbarg \& Tudinger, 1998), we set $V(z)=e^{2 d}-e^{d r^{\beta}}, z \in \Omega_{0}$, in which the constants $d>0$ and $\beta<0$ will be determined later. A direct computation yields

$$
\begin{aligned}
& \Delta V-\sigma \sum_{i=1}^{2} \sum_{j=1}^{2} a_{i j}(z) \partial_{i j}^{2} V-\sigma \sum_{i=1}^{2} b_{i}(z) \partial_{i} V \\
&=-(\beta d) r^{\beta-2} e^{d r^{\beta}}\left(\beta d r^{\beta}+\beta\right)+\sigma A^{-1}(\beta d) r^{\beta-4} e^{d r^{\beta}}+\frac{2 \sigma(\beta d) r^{\beta-4} e^{d r^{\beta}}}{A-\frac{1}{r^{2}}} \\
& \quad<-(\beta d)^{2} r^{2 \beta-2} e^{d r^{\beta}}
\end{aligned}
$$

Notice that $\left|\frac{1}{A \rho_{0}^{2}(r)} F_{1}(z, \nabla \Phi, \Psi)\right| \leq C_{1}\left|F_{1}(z, \nabla \Phi, \Psi)\right|_{0,0}^{(3)} r^{-3}$, where $C_{1}>0$ is dependent of $A$ and $B$. we can choose $\beta=-\frac{1}{2}$ and set $\hat{V}_{l}(z)=\left(\left|F_{1}(z, \nabla \Phi, \Psi)\right|_{0,0}^{(3)}+l^{-2} \sigma\left|\nabla \Phi_{\sigma}\right|_{1,0}^{(1)}\right) V(z)$ for any $l>1$. Then there exists a suitably large constant $d>0$ which is independent of $\sigma, \delta, \varepsilon$ and $l$ such that

$$
\begin{gathered}
L_{\sigma}\left(\hat{V}_{l}(z)-\Phi_{\sigma}\right)<0, \quad \text { in } \quad \Omega_{0}, \\
\hat{V}_{l}(z)-\Phi_{\sigma}>0, \quad \text { on } \quad|z|=1, \quad \hat{V}_{l}(z)-\Phi_{\sigma}>0, \quad \text { as } \quad|z| \rightarrow+\infty .
\end{gathered}
$$

here the $L^{\infty}$ estimates (28) play the key poles.

Then it follows the maximum principle in (Gilbarg \& Tudinger, 1998) that

$$
\left|\Phi_{\sigma}\right|_{0,0}^{(0)} \leq e^{2 d}\left(\left|F_{1}(z, \nabla \Phi, \Psi)\right|_{0,0}^{(3)}+l^{-2} \sigma\left|\nabla \Phi_{\sigma}\right|_{1,0}^{(1)}\right) .
$$

From Lemma 6.20 in (Gilbarg \& Tudinger, 1998) together with (30) and the estimate (28), we have

$$
\left|\Phi_{\sigma}\right|_{2, \alpha}^{(0)} \leq C\left(\left|\Phi_{\sigma}\right|_{0,0}^{(0)}+\left|F_{1}(z, \nabla \Phi, \Psi)\right|_{0, \alpha}^{(2)}\right) \leq C\left(\left|F_{1}(z, \nabla \Phi, \Psi)\right|_{0, \alpha}^{(3)}+l^{-2} \sigma\left|\nabla \Phi_{\sigma}\right|_{1,0}^{(1)}\right) .
$$

and

$$
\begin{gathered}
\left|\nabla \Phi_{\sigma}\right|_{1, \alpha}^{(2)} \leq C\left(\left|\Phi_{\sigma}+\int_{1}^{\infty} e^{-\int_{1}^{r} c_{3}(s) d s} \int_{r}^{\infty} e^{\int_{1}^{l} c_{3}(s) d s} f_{0}(l) d l d r\right|_{0,0}^{(1)}+\left|F_{1}(z, \nabla \Phi, \Psi)\right|_{0, \alpha}^{(3)}\right) \\
\leq C\left(\left|F_{1}(z, \nabla \Phi, \Psi)\right|_{0, \alpha}^{(3)}+\sigma\left|\nabla \Phi_{\sigma}\right|_{1,0}^{(1)} r^{-1}\right) .
\end{gathered}
$$

Connecting (31)-(32) with the second equation in (19) and setting $l>1$ large enough, then we have

$$
\left|\Phi_{\sigma}\right|_{2, \alpha}^{(0)}+\left|\nabla \Phi_{\sigma}\right|_{1, \alpha}^{(2)}+\left|\Psi_{\sigma}\right|_{1, \alpha}^{(3)} \leq C\left(\left|F_{1}(z, \nabla \Phi, \Psi)\right|_{0, \alpha}^{(3)}+\left|F_{2}(z, \nabla \Phi, \Psi)\right|_{1, \alpha}^{(3)}\right) .
$$

Thus we have completed the proof of Lemma 3.2. 
Lemma 3.3(Cui \& Li, 2011) For each $F(z) \in H_{0, \alpha}^{(3)}\left(\Omega_{0}\right)$, the following problem

$$
\left\{\begin{array}{l}
\Delta \varphi=F(z), \quad \text { in } \Omega_{0}, \\
\varphi=0, \text { on }|z|=1, \\
\lim _{|z| \rightarrow \infty} \frac{\varphi(z)}{\ln |z|}=0,
\end{array}\right.
$$

has a unique solution $\varphi \in C^{2, \alpha}\left(\bar{\Omega}_{0}\right)$ satisfying

$$
|\varphi|_{2, \alpha}^{(0)}+|\nabla \varphi|_{1, \alpha}^{(2)} \leq C|F|_{0, \alpha}^{(3)} .
$$

Proof of Theorem 2.1. Since the uniform weighted Hölder estimate (20) for any $\sigma \in[0,1]$ is derived, the existence of the solution to the linearized problem of (15) can be solved by the continuity method. Furthermore, using the standard fixed point argument, one can derive the existence and uniqueness of the solution of nonlinear problem (15).

Firstly, by Lemma 3.3 together with the uniform weighted Hölder estimate (20) and the continuity method in ( Gilbarg \& Tudinger, 1998), we know that for any $(\hat{\Phi}, \hat{\Psi})$, if $|\nabla \hat{\Phi}|_{1, \alpha}^{(2)}+|\hat{\Psi}|_{1, \alpha}^{(3)}$ is bounded, the linearized problem

$$
\left\{\begin{array}{l}
L_{1}(\Phi)=\Delta \Phi-\left(\sum_{i=1}^{2} \sum_{j=1}^{2} a_{i j}(z) \partial_{i j}^{2} \Phi+\sum_{i=1}^{2} b_{i}(z) \partial_{i} \Phi\right)=-\frac{1}{A \rho_{0}^{2}(r)} F_{1}(z, \nabla \hat{\Phi}, \hat{\Psi}), \quad \text { in } \Omega_{0}, \\
\Psi=\frac{1}{\Pi(z)} \partial_{1} \phi_{0} \partial_{1} \Phi+\frac{1}{\Pi(z)} \partial_{2} \phi_{0} \partial_{2} \Phi+F_{2}(z, \nabla \hat{\Phi}, \hat{\Psi}), \quad \text { in } \Omega_{0}, \\
\Phi=0, \text { on }|z|=1, \\
\lim _{|z| \rightarrow \infty} \frac{\Phi(z)}{\ln |z|}=0,
\end{array}\right.
$$

has a unique solution $(\Phi, \Psi)$ with the estimate

$$
|\Phi|_{2, \alpha}^{(0)}+|\nabla \Phi|_{1, \alpha}^{(2)}+|\Psi|_{1, \alpha}^{(3)} \leq C\left(\left|F_{1}(z, \nabla \hat{\Phi}, \hat{\Psi})\right|_{0, \alpha}^{(3)}+\left|F_{2}(z, \nabla \hat{\Phi}, \hat{\Psi})\right|_{1, \alpha}^{(3)}\right) .
$$

We now construct a mapping as in (Cui \& Li, 2011): $T(\hat{\Phi}, \hat{\Psi})=(\Phi, \Psi)$. For any two $\left(\hat{\Phi}_{i}, \hat{\Psi}_{i}\right)(i=1,2)$ satisfying $\left|\nabla \hat{\Phi}_{i}\right|_{1, \alpha}^{(2)}+\left|\hat{\Psi}_{i}\right|_{1, \alpha}^{(3)}<\delta$ with $\delta>0$ to be determined, then the problem (34) have two corresponding solutions $\left(\Phi_{i}, \Psi_{i}\right)(i=1,2)$ respectively. In addition, according to Lemma 3.1 and Lemma 3.2, we have

$$
\left|\Phi_{i}\right|_{2, \alpha}^{(0)}+\left|\nabla \Phi_{i}\right|_{1, \alpha}^{(2)}+\left|\Psi_{i}\right|_{1, \alpha}^{(3)} \leq C\left(\left|F_{1}\left(z, \nabla \hat{\Phi}_{i}, \hat{\Psi}_{i}\right)\right|_{1, \alpha}^{(3)}+\left|F_{2}\left(z, \nabla \hat{\Phi}_{i}, \hat{\Psi}_{i}\right)\right|_{0, \alpha}^{(3)}\right) \leq C\left(\varepsilon+\delta^{2}\right),
$$

where the generic constant $C$ is independent of $\varepsilon$ and $\delta$.

Let $\delta=\frac{1}{1+3 C}$ and $\varepsilon_{0}=\delta^{2}$, then for $0<\varepsilon<\varepsilon_{0}$, we get $\left|\Phi_{i}\right|_{2, \alpha}^{(0)}+\left|\nabla \Phi_{i}\right|_{1, \alpha}^{(2)}+\left|\Psi_{i}\right|_{1, \alpha}^{(3)}<\delta$. This implies that $T$ is a continuous mapping from $\Xi_{\delta}=\left\{(\Phi, \Psi):|\nabla \Phi|_{1, \alpha}^{(2)}+|\Psi|_{1, \alpha}^{(3)}<\delta,\left.\Phi\right|_{|z|=1}=0, \lim _{|z| \rightarrow \infty} \frac{\Phi(z)}{\ln |z|}=0\right\}$ into itself. By (35) together with Lemma 3.1, we also have the estimate

$$
\begin{aligned}
&\left|\Phi_{1}-\Phi_{2}\right|_{2, \alpha}^{(0)}+\left|\nabla\left(\Phi_{1}-\Phi_{2}\right)\right|_{1, \alpha}^{(2)}+\left|\Psi_{1}-\Psi_{2}\right|_{1, \alpha}^{(3)} \\
& \quad \leq C(\varepsilon+\delta)\left(\left|\nabla\left(\hat{\Phi}_{1}-\hat{\Phi}_{2}\right)\right|_{1, \alpha}^{(2)}+\left|\hat{\Psi}_{1}-\hat{\Psi}_{2}\right|_{1, \alpha}^{(3)}\right) \leq \frac{4}{5}\left(\left|\nabla\left(\hat{\Phi}_{1}-\hat{\Phi}_{2}\right)\right|_{1, \alpha}^{(2)}+\left|\hat{\Psi}_{1}-\hat{\Psi}_{2}\right|_{1, \alpha}^{(3)}\right) .
\end{aligned}
$$

This implies that $T$ is contractible in $\Xi_{\delta}$ and the nonlinear problem (15) has a unique solution $(\Phi, \psi) \in \Xi_{\delta}$ which satisfies

$$
|\Phi|_{2, \alpha}^{(0)}+|\nabla \Phi|_{1, \alpha}^{(2)}+|\Psi|_{1, \alpha}^{(3)} \leq C \varepsilon
$$

Thus Theorem 2.1 is proved. By the inverse of the coordinate $z_{1}=x_{1}(1+\delta(x))^{-1}, z_{2}=x_{2}(1+\delta(x))^{-1}$ and the assumption that $\delta(x)$ has compact support, then the proof of Theorem 1.1 is obvious.

\section{Acknowledgements}

This work was supported by Jiangsu Province University Natural Science Research Project (No.14KJB110018). The author thanks the anonymous referees for their valuable comments and suggestions.

\section{References}

Bers, L. (1945). On the circulatory subsonic flow of a compressible fluid past a circular cylinder. Tech. Notes Nat. Adv. Comm. Aeronaut, 970, 30pp.

Bers, L. (1958). Mathematical aspects of subsonic and transonic gas dynamics. Surveys in Applied Mathematics, 3. New York, NY: John Wiley \& Sons, Inc.; London, England: Chapman \& Hall, Ltd. 
Chen, J. (2009). Subsonic flow for the full Euler equations in half plane. J. Hyperbolic Differ. Equ., 6, 207-228. https://doi.org/10.1142/S0219891609001873

Courrant, R., \& Friedrichs, K. O. (1948). Supersonic flow and shock waves. New York, NY: Interscience Publishers Inc.

Cui, D. C., \& Li, J. (2011). On the existence and stability of 2-D perturbed steady subsonic circulatory flows. SCIENCE CHINA Mathematics, 54, 1421-1436. https://doi.org/10.1007/s11425-011-4226-5

Cui, D. C., \& Yin, H. C. (2007). Global conic shock wave for the steady supersonic flow past a cone: isothermal case. Pacific Journal of Mathematics, 233, 257-289. https://msp.org/pjm/2007/233-2/p01.xhtml

Dong, G. C., \& Ou, B. (1993). Subsonic flows around a body in space. Comm. P. D. E., 18, 355-379.

Finn, R., \& Gilbarg, D. (1957). Asymptotic behavior and uniqueness of plane subsonic flows. Comm.Pure Appl.Math., $10,23-63$.

Gilbarg, D., \& Tudinger, N. S. (1998). Elliptic partial differential equations of second order, Second edition. Grundlehren der Mathematischen Wissenschaften, 224. Berlin-New York: Springer.

Li, L., Xu, G., \& Yin, H. C. (2015). On the instability problem of a 3-D transonic oblique shock wave. Advances in Mathematics, 282, 443-515. https://doi.org/10.1016/j.aim.2015.06.018

Thwaites, B. (1950). Note on the circulatory flow about a circular cylinder through which the normal velocity is large. Quart. J. Mech. Appl. Math., 3, 74-79.

\section{Appendix}

In this appendix, we will construct the background solutions (8) for the nonlinear problem (7) in the exterior domain $\Omega_{0}=\{x:|x|>1\}$. As illustrated in section 104 in (Courant \& Friedrichs, 1948), we know the the streamlines of the circulatory flow in the domain $\Omega_{0}$ are constituted with circles with the center at the origin. So we introduce the polar coordinate transformation $x_{1}=r \cos \theta, x_{2}=r \sin \theta$, and decompose $\left(u_{10}, u_{20}\right)$ as $u_{10}=U_{10} \cos \theta-U_{20} \sin \theta, u_{20}=$ $U_{10} \sin \theta+U_{20} \cos \theta$. As in (Cui \& Li, 2011) the system (1)-(5) can be written as

$$
\left\{\begin{array}{l}
\partial_{r}\left(\rho_{0} U_{10}\right)+\frac{1}{r} \partial_{\theta}\left(\rho_{0} U_{20}\right)=0 \\
\partial_{r} U_{20}+\frac{1}{r} U_{20}=0 \\
\frac{1}{2}\left|U_{20}\right|^{2}+A \ln \rho_{0}=B \\
U_{10} \equiv 0
\end{array}\right.
$$

and

$$
\lim _{r \rightarrow \infty} \frac{1}{\ln r} \int_{1}^{r}\left(\rho_{0} U_{20}\right)(s, \theta) d s=e^{\frac{B}{A}} .
$$

It follows from (36) that

$$
\rho_{0}=\rho_{0}(r), \quad U_{20}=\frac{\kappa}{r}, \quad \frac{\kappa^{2}}{2 r^{2}}+A \ln \rho_{0}(r)=B,
$$

where $k$ is some constant to be determined.

$$
\rho_{0}(r)=\exp \left(\frac{1}{A}\left(B-\frac{\kappa^{2}}{2 r^{2}}\right)\right)
$$

Moreover, by the definition of stream function in (6), the corresponding stream function $\psi_{0}(r)$ satisfies

$$
\psi_{0}(r)=\int_{1}^{r} \rho_{0}(s) \frac{\kappa}{s} d s+m
$$

Combining (37) with (40), we have

$$
\lim _{r \rightarrow \infty} \frac{\psi_{0}(r)}{\ln r}=\lim _{r \rightarrow \infty} r \rho_{0}(r) \frac{K}{r}=e^{\frac{B}{A}} .
$$

This together with (39) yields $\kappa \equiv 1,(8)$ is arrived at.

\section{Copyrights}

Copyright for this article is retained by the author(s), with first publication rights granted to the journal.

This is an open-access article distributed under the terms and conditions of the Creative Commons Attribution license (http://creativecommons.org/licenses/by/4.0/). 